\title{
A preference for progressivity in interaction
}

\author{
TA N Y A S T V E R S \\ Max Planck Institute for Psycholinguistics \\ PB 310 \\ NL-6500 AH Nijmegen, The Netherlands \\ Tanya.Stivers@mpi.nl \\ J E F F R E Y D. ROB I N S O N \\ Department of Communication \\ Rutgers University \\ 4 Huntington Street \\ New Brunswick, NJ 08901 \\ jrob@scils.rutgers.edu
}

\section{A B S T R AC T}

This article investigates two types of preference organization in interaction: in response to a question that selects a next speaker in multi-party interaction, the preference for answers over non-answer responses as a category of a response; and the preference for selected next speakers to respond. It is asserted that the turn allocation rule specified by Sacks, Schegloff \& Jefferson (1974) which states that a response is relevant by the selected next speaker at the transition relevance place is affected by these two preferences once beyond a normal transition space. It is argued that a "second-order" organization is present such that interactants prioritize a preference for answers over a preference for a response by the selected next speaker. This analysis reveals an observable preference for progressivity in interaction. (Interaction, conversation analysis, preference, sequence organization.)*

\section{N T R O D U C T I O N}

Sequences such as the following can be observed in spontaneous, naturally occurring social interaction (Stivers 2001, Lerner 2002):

(1) Fish Dinner: 16:00

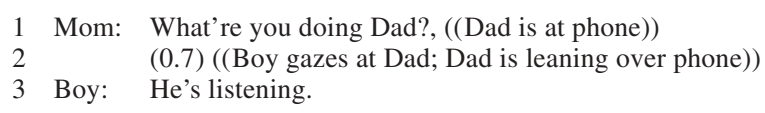

Here a family is having dinner. The mother selects the father to respond to a question by using an address term, Dad. However, it is their son who actually answers the question. Most of the time in question sequences, the selected next speaker provides an answer in response to the question, so next-speaker selection and answering are intertwined. This article treats such sequences as a locus 
for examining what happens when these two normally intertwined interactional outcomes are separated and therefore can be observably "ranked" in terms of their interactional importance by and for interactants. In particular, examples such as this pit the relevance of an answer to a question (as a category of response) against the relevance of that response's being provided by the selected next speaker. Each of these issues has its own structural preferences and organization, and we will rely on these in order to examine the organization of such preferences with respect to one another, and what this organization reveals about the structure of social interaction.

\section{B A C K G R O U N D}

This article builds on earlier work focused on pediatric encounters involving parents, children, and physicians. In that context, it was observed that physicians often selected children to respond to questions, but parents did most of the answering (Stivers 2001). The article argued that parents did not answer questions for which their children had been selected immediately at the transition relevance place, but only when some type of "interactional difficulties" emerged. Such difficulties took the form of silence, non-answer responses, or other displays of difficulty in answering the question. The present article begins with the question of whether the practice of non-selected recipients responding to questions that selected a co-interactant is a more general interactional phenomenon, and it seeks to explain the underlying mechanisms governing this domain.

The concept of preference with respect to responsive actions is rooted in the observations of Schegloff and Sacks (Sacks 1967; Schegloff 1972; Schegloff \& Sacks 1973) that, in adjacency pair sequences - of which the question-answer sequence is a prototypical example - respondents to the first pair part action routinely have available two or more normatively possible, though nonequivalent, responses. Analyzing both the position of the response turn (e.g., whether it is delayed) and its design (e.g., whether it includes an account for the response or just the response), makes is possible to understand certain responses as "preferred" in a structural (not a psychological) sense, and other responses as "dispreferred" (Sacks 1973a, 1987a; Schegloff, Jefferson, \& Sacks 1977; Pomerantz 1978, 1984a; Atkinson \& Heritage 1984; Heritage 1984, 1988; Clayman 1993, 2002; Golato 2002; Raymond 2003). For instance, when a question that prefers agreement receives agreement, these sequences tend to be accomplished more quickly, without internal delays and without extended accounts (Sacks 1973b, 1987a; Heritage 1984). In an introduction to a series of chapters on preference organization, Atkinson \& Heritage (1984:55) state that "the institutionalized design features of preferred/dispreferred actions are both inherently structured and actively used so as to maximize cooperation and affiliation and to minimize conflict in conversational activities." Thus, the concept of preference is important to 
our understanding not only of how members of a society communicate, but also to the larger issue of social organization (Heritage 1984, Clayman 2002).

This article is concerned with two fundamental aspects of preference revolving around question-answer adjacency pairs. First, as Schegloff \& Sacks 1973 observe, an answer is conditionally relevant after a question. However, there are two primary ways in which the requirement of conditional relevance can be satisfied. First, a recipient can provide an answer. Second, as Heritage 1984 discusses, a recipient can provide a non-answer response which addresses the relevance of an answer, typically by providing an account for not answering. Although two categories of response are thus possible upon the completion of a question, these response categories are nonequivalent and thus can be ranked in terms of preference. Evidence for this will form the basis for the first section of this article.

In working through this analysis, it can further be observed that most questions in multi-party interaction select a next speaker to provide the answer. Sacks, Schegloff, \& Jefferson (1974:704) state that the first rule of turn allocation is that, at a transition-relevance place, "If the turn-so-far is so constructed as to involve the use of a 'current speaker selects next' technique, then the party so selected has the right and is obliged to take next turn to speak; no others have such rights or obligations, and transfer occurs at that place." However, there are times when responses from non-selected recipients are provided and are not treated as violations of the turn-taking system (Stivers 2001). ${ }^{1}$ This article will show that after a transition relevance place, who responds is governed not by conditional relevance but by preference. The second section will examine this second preference.

Having documented preferences both for answers and for selected next speakers to respond, we turn to a third issue: Can these preferences be ranked relative to each other? Is there a second order of organization that can be seen to govern situations where both preferences cannot be satisfied simultaneously? Although much of the research on preference organization has focused on discrete preferences, some work has examined occasions where "multiple constraints" (Pomerantz 1978) or "two preferences" (Sacks \& Schegloff 1979) cannot be satisfied simultaneously. Sacks \& Schegloff argued that an examination of such materials both gives support to the proposal that separate preferences are involved and permits "the extraction of a 'second order' organization directed to an INTEGRATION of preferences on occasions where their potential concurrent satisfiability is not realized" (Sacks \& Schegloff 1979:16). There is one context in particular where the preference for an answer and the preference for the selected next speaker to respond can come into conflict, and that is when a question selects a next speaker in a multi-party context, and that selected next speaker does not provide an answer immediately upon completion of the question. In such contexts, interactants are faced with the problem of which preference to prioritize. It is this issue with which the final section of this article is concerned. 
The data examined here are video recordings of naturally occurring interactions among friends and family in a range of different settings, and of doctor-patient encounters. All of the data are in American English. The range of data supports the argument that this practice is not restricted to a particular context or particular speakers, but rather occurs in a variety of communication contexts and among a variety of speakers.

The data have been transcribed (see Appendix A) and analyzed using Conversation Analysis (for a review see Heritage 1984). In line with this methodology, we made a collection of approximately 260 instances of question sequences in multi-party interaction and qualitatively examined similarities and differences among the collected instances. We focused on information questions because other types of questions (such as requests for action) are far more complicated in terms of preference organization. Of course, information questions can simultaneously be vehicles for doing actions other than requesting information, but our procedure enabled us to determine the boundaries of the practice. The cases shown here are representative of the cases in the collection but were selected as particularly clear examples of the phenomenon, although they are not qualitatively different from other instances in the collection. In addition, to examine deviant cases more carefully, we examined further data to extract additional instances for analysis. A subset of these cases is shown in the discussion of when nonselected recipients respond to questions which had selected another as next speaker.

\section{A N A L Y S I S}

As Schegloff \& Sacks 1973 write, an adjacency pair is composed of two pairtyped parts, with the second part due upon completion of the first. The normativity of this structure is evidenced in three ways: (i) first pair parts (FPPs) are regularly responded to with second pair parts (SPPs); (ii) participants work in various ways to display an orientation to the relevance of a SPP when one is not immediately forthcoming (this will be discussed more in the section on nonanswer responses); and (iii) when a SPP is not forthcoming, speakers may sanction the participant from whom this was due. The following is an example of a simple information question-answer adjacency pair:

(2) Fish Dinner: 9:34

1 Boy: What kind of fish is (it)/(this).

2 Mom: .h Halibut,

Here the request for information makes conditionally relevant an answer which is provided at line 2. Another example: 
(3) Housemates 22:56

1 Jud: Why is that one on.

2 Lan: B'cause I turned it on.

Here too a request for information makes relevant an answer, and that is provided in line 3 . In a third example (4), a grandmother and granddaughter are talking on the phone. The grandmother asks a question (line 6) which is not responded to (line 7). This is sanctioned through pursuit of an answer (line 8):

(4) Beach

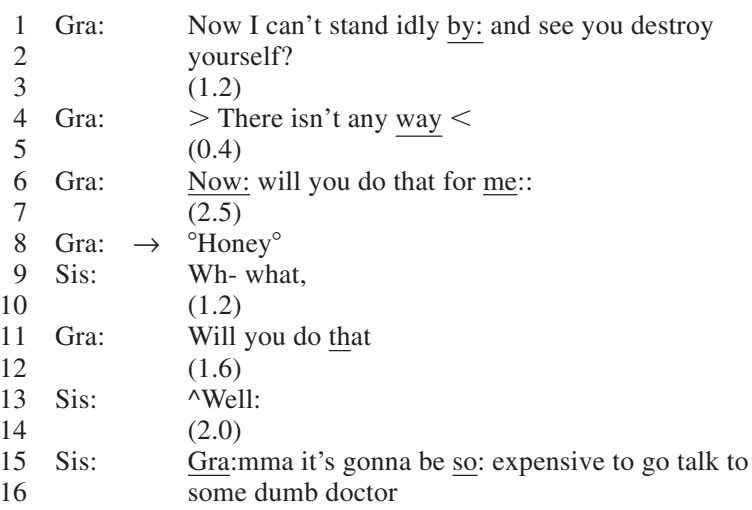

The pursuit of an answer, though it requires a redoing of the question (line 11), is ultimately successful in eliciting a response to the question. One of the most fundamental observations about these adjacency pairs is that questions make answers relevant. That said, the principle of conditional relevance can be satisfied by answers (actions that further the progress of the activity), and by non-answer responses that either 1) display an orientation to the relevance of an answer but satisfy only the technical two-part structure of a sequence (e.g., I don't know) or 2) that impede the progress of the sequence (e.g., initiations of repair or counters). Thus, although a non-answer response is a normatively viable action in response to a question, it is a dispreferred alternative.

\section{Preference for an answer response}

The evidence that an answer is the alternative preferred over a non-answer response takes several forms. ${ }^{2}$ First, answers are the most common category of response to information questions. Second, the turn shapes of answers and nonanswer responses reflect their ranking as preferred and dispreferred categories of response. Third, interactants typically treat non-response as indicating disalignment rather than indicating that no response will be forthcoming. Fourth, speakers do interactional WORK to provide answers, despite non-answers being a readily available alternative category of response. 
Regularity of answer responses. Answers such as those shown in (2-5) are the most frequent category of responses to questions. In approximately $85 \%$ of the cases in the present collection, the selected next speaker provides an answer to the question.

Non-answer response turn shapes. Non-answer responses are delivered in a manner that reflects "an institutionalized ranking of alternatives" (Atkinson \& Heritage 1984) in that they are frequently (i) delayed both within and between turns, (ii) prefaced by vocal markers (e.g., Uh or Well), and (iii) expanded with accounts (Heritage 1984, Pomerantz 1984a, Sacks 1987a). For an example, see (5). In line 1, Maggie asks an information question. An answer is not provided at first possible completion (the transition relevance place at the end of line 1). Maggie then pursues uptake with a replacement and respecification of work in line 2. At the completion of the new addition to the turn, response is once again due. At this point Christina provides a non-answer response (line 3):

(5) Trio 2

1 Mag: What happened at (.) wo:rk.

2 Mag: At Bullock's this evening.

3 Chr: .hh Wul I don' kno:^::w.

Christina's response is delayed both between the question and Christina's answer (with the inbreath.$h h$ ) and within the response turn with the well preface and further with the prosodic elongation of $k n o:^{\wedge}:: w$. Here, whereas an answer about what had happened would further the activity of informing, the nonanswer response does not further the activity even though it completes the sequence.

Non-answer responses such as this are, in themselves, often accounts for not answering the question to which they are responding. In (5), the account is that Christina does not know the answer - a claim of inability to answer the question (Heritage 1984, Beach \& Metzger 1997). However, often non-answer responses are still further accounted for, as below:

(6) Chicken Dinner 1:33

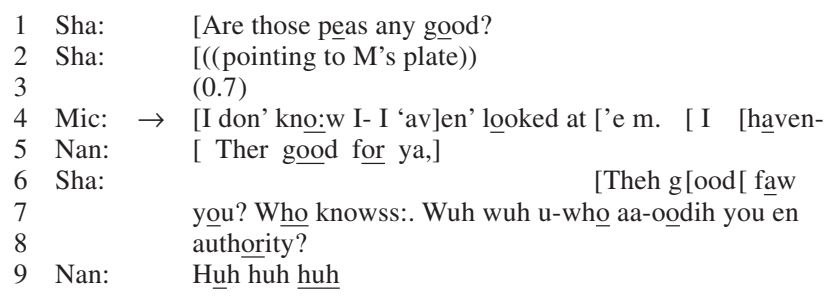

Michael's response to Shane's question accounts for not answering the question (line 1) by claiming an inability to answer the question. Subsequently, he expands his response by offering an account for why he does not know: because 
he has not tried them (or as he puts it, looked at 'em). Thus, the dispreferred $I$ don' kno: $w$ is itself accounted for, which is characteristic of dispreferred turn shapes (Pomerantz 1978, 1984a; Heritage 1984; Sacks 1987a).

Accounts reveal what is potentially problematic about non-answer responses: Although they address the relevance of a response to the question, non-answer responses fail to collaborate with promoting the progress of the activity through the sequence. In this way they satisfy only the technical structural aspect of sequence closure while failing to promote closure of the activity.

Speakers treat non-response as disaligning. A third type of evidence that answers are the preferred category of response is that, in the absence of an immediate response to a question, prior speakers typically treat this silence as indicating disalignment with the question and not either as projecting no response or as projecting a non-answer response. Sacks (1973b, 1987a, 1987b) and Pomerantz (1984b) discuss this more fully in terms of questioners who revise the polarity of the question such that an answer that would have been disaligning with the design of the initial question becomes aligned with the design of the revised question. An example of this follows:

(7) Housemates 8:30

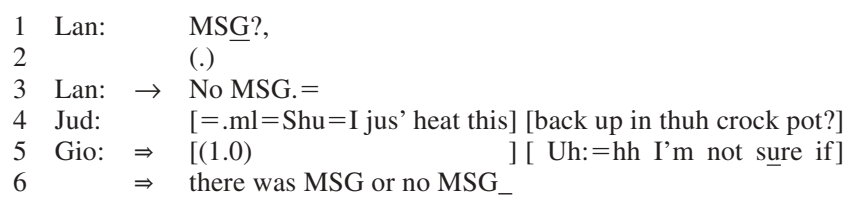

Lance and Gio have been discussing Gio's lunch. Lance asks whether Gio's meal had MSG in it (a chemical that is often found in Chinese foods). He asks this (line 1) in such a way that it reaches hearable intonational and pragmatic completion. As a declarative construction in Lance's domain of experience, it makes confirmation or disconfirmation relevant. Confirmation is preferred because this would align with the candidate response proposed with $M S \underline{G}$ ? When a response is not immediately forthcoming (line 2), Lance reverses the polarity of the question so that a "no" answer would now be in alignment with the question (line 3). Note that by reversing the polarity of the question, Lance treats Gio's silence at line 2 as being preliminary to non-alignment. Instead, Gio ultimately responds that he is unable to answer the question because he is not sure (lines 5-6). Further note that, similar to the cases shown earlier, here too the non-answer response is delivered after between-turn delay (lines 2 and 5) and within-turn delay (with the $U h$ preface, the stretch on $U h$ :, and the outbreath $h h$ ). Thus, although disagreeing/non-aligned answers are themselves dispreferred, a non-aligned ANSWER is still treated as preferred over a NONANSWER response. 
Participants work to receive and provide answers. A fourth type of evidence that answers are preferred over non-answer responses can be seen in the way that participants struggle to receive and provide answers if at all possible. For instance, returning to (7), Lance's reverse in polarity is specifically an effort to elicit an answer from Gio. Note in particular that, even if that answer will be disaligned with the initial question design, the pressure for an answer is such that speakers will typically redesign their question in order to get an answer (Pomerantz 1984b, Sacks 1987a).

Moreover, it is not only questioners who do work to secure an answer as a SPP. Question recipients also do work to provide answers, even when they are not truly ABLE to provide a definitive answer. For instance, see (8). Here, Judy, Gio, and Robert are preparing dinner together. Judy asks Gio whether he thinks the French fries they are preparing are done. Gio does work to provide an answer, despite the fact that I don't know is, in principle, an available response. He is across the kitchen from the stove and thus cannot see the fries without moving and thus cannot assess whether they are done. However, when Judy asks a question, Gio does what most interactants do: He works to provide an answer. He moves across the kitchen to look at the fries and then provides an answer.

(8) Housemates 27:30 ((Judy, Gio, and Robert are in the kitchen))

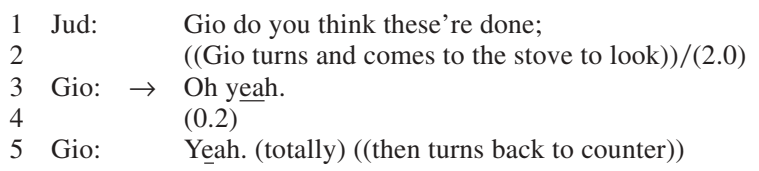

Speakers also work to provide answers to questions even when they are in no position truly to answer them. For instance:

(9) SBL:2.2 (analyzed by Pomerantz 1984a:58)

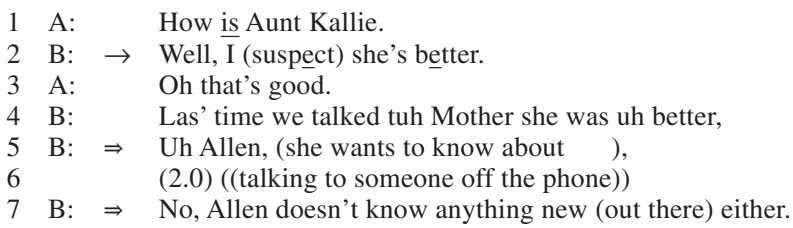

Here, A asks B a question which makes relevant an assessment of Kallie. In response, B provides an answer. However, note that her answer is prefaced by Well and is mitigated with I suspect rather than providing a straightforward assessment and answer. This is then accounted for first with the evidence that she (and presumably Allen) has that Aunt Kallie is, in fact, better - she was better the last time they inquired about her (line 4). Second, she provides an account, though rather obliquely, for her failure to provide a more unequivocal assessment/ answer: She does not know (as carried by the either in line 7). Finally, note that B asks another interlocutor, Allen, if he can provide an answer (line 5), and when 
he cannot, this is also accounted for (line 7). Important to observe here is that B works hard to provide an answer to A's question despite I don't know being an available alternative response. In the end, this leaks out through the either in line 7, but it is never provided as an overt on-record response. A similar case is shown in (10):

(10) FD:1 (taken from Pomerantz 1984a:59)

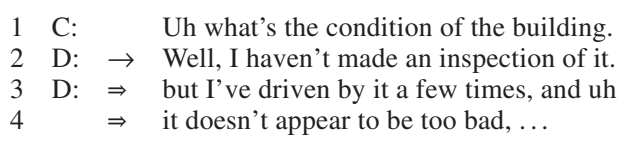

Here, following C's question, which makes relevant an assessment of the building, D provides an account for why he cannot provide a definitive answer to the question: He has not inspected it (line 2). However, he then goes on nonetheless to provide an answer that is epistemically qualified with appear. Here D does work to answer the question even though it appears that in a sense he doesn't really know the answer. ${ }^{3}$

Extracts (7-10) all provide evidence that interactants work to secure answer responses whenever possible. In this way, both speakers and hearers display a concern not only with the conditional relevance of sequence closure but also with the progress of the activity for which the sequence was a vehicle. Thus, answers may be seen as legitimizing and furthering the progress of the ongoing activity. To summarize, answers and non-answer responses are, to use Atkinson \& Heritage's (1984) terms, alternative but nonequivalent categories of response which can be ranked. The evidence provided here supports the analysis that an answer is the category of response preferred over a non-answer response - independent of the alignment of that answer to the design of the question.

\section{Preference for selected next speakers to respond}

Having established, at least initially, that answers are the preferred category of response following a question, let us now turn to a second fundamental preference: Among questions that select a next speaker, there is a preference for the selected next speaker rather than another interactant to respond to the question. Here the invocation of the term "non-selected recipient" becomes relevant. This is meant to refer to interactants who are participants in the ongoing sequence but were not otherwise selected to respond to the immediately prior question. "Nonselected next speakers" refers to the same category of persons, but this label can be used only after they self-select and become "next speakers."

The conditional relevance of a response by the selected next speaker. Although not all questions select a next speaker in multi-party conversation, most of the time they do appear to, whether explicitly by gaze or by an address term (Sacks et al. 1974), or tacitly by context-sensitive means (Lerner 2003). When speakers do select a next speaker, the first turn allocation rule specifies that the 
selected speaker has the right and obligation to speak next (Sacks et al. 1974). Data support that this rule is oriented to by participants AT THE TRANSITION RELEVANCE PLACE in three main ways: (i) Selected speakers routinely begin responding at the transition relevance place; (ii) non-selected recipients, when they do respond, generally restrict their responses to answers; and (iii) nonselected recipients, even when they are otherwise able to answer a question, rarely do so AT the transition relevance place. This section will explore each of these in turn. $^{4}$

Regularity of responses by selected next speakers. In the current collection of question sequences, $97 \%$ of the time selected next speakers responded to questions. In the remainder of cases, non-selected recipients generally responded and did so by answering the question in nearly all cases. Although reliable ethnographic evidence does not exist for each case, it can be seen that often, because the participants were close friends and family members, multiple interactants were "in the know" and could have supplied the answer but did not.

Response types: selected vs. non-selected next speaker. Whereas non-selected recipients' responses were almost exclusively concerned with the activity with which the question sequence was concerned and thus with answering the question, selected next speakers, in approximately $12 \%$ of cases, responded with something other than an answer. I don't know-type responses were the most common type of non-answer response, but it was not uncommon for selected next speakers to initiate new sequences with a repair initiation or a counter (Schegloff 1972). ${ }^{5}$ For instance, see (11). Here, three housemates are preparing dinner together. After Lance has declined a beverage from Gio, Judy challenges this with a question, Why not, in line 10. In response, she receives not an answer but rather another question, which challenges the terms of her own question because it treats the question as implying that Lance sHould have a beverage.

(11) Housemates 03:05

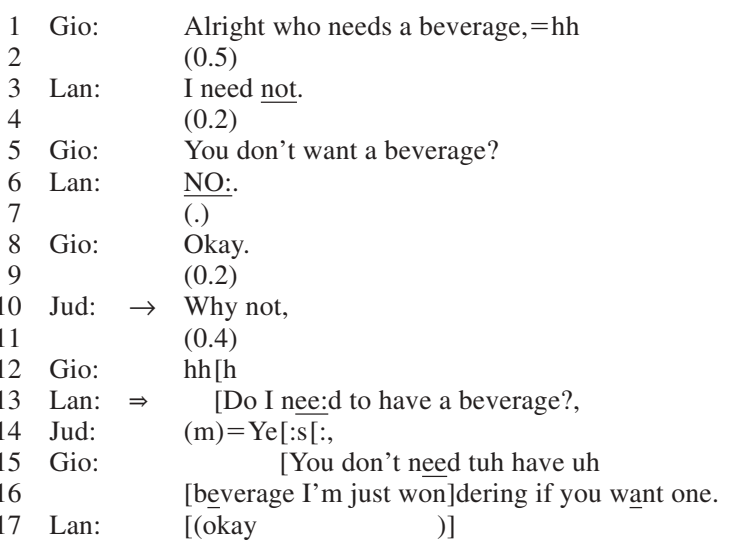


Another example is shown in (12). Here, two housemates are talking. Tara has indicated that she started crying when talking to an ex-boyfriend on the phone. Kristina requests confirmation of her understanding in line 2. Following the question, Tara, the selected next speaker, initiates repair in line 3 with What?:

(12) SB $139: 30$

$\begin{array}{llll}1 & \text { ???: } & & {[((\text { other conversations going on }))} \\ 2 & \text { Kri: } & & \text { [You cried tuh him on thuh pho:ne? } \\ 3 & \text { Tar: } \rightarrow & \text { What? } \\ 4 & \text { Kri: } & & \text { You cried to him on the phone? } \\ 5 & \text { Tar: } & \text { Not on } \mathrm{p}(\mathrm{h}) \text { urpose_ }\end{array}$

Whereas actions such as this are easily understood by interlocutors to be on their way to addressing the question (Schegloff 1972, in press), non-selected recipients are not observed to perform this same range of actions following questions. Non-selected recipients appear generally to restrict their responses to answers. This suggests that there is a preference for selected next speakers to respond to questions, and that selected next speakers display an orientation to this preference as discrete from the preference for an answer, in that they will perform other sorts of responsive actions following information questions.

Non-selected recipients withhold response at the transition relevance place. A third type of evidence that there is a preference for selected next speakers to respond to questions that select them is that, even when a non-selected recipient can be seen to be "in the know" and thus able to answer the question, he or she rarely does so immediately following the question (i.e., at the TRP). This was first documented in the pediatric environment (Stivers 2001). There one might expect that parents would be rather quick to speak on behalf of their children, especially very young children. However, this was not the case. Even with children who are 2 or 3 years of age, non-selected recipients who are "in the know" (i.e., they made the appointments for the children and brought them to the doctor) withhold response at the TRP. This appears to be a much more general phenomenon, as documented here.

For instance, see (13). Here several housemates are preparing dinner together. Judy is cooking French fries on the stove and selects Gio to answer a question about whether they are done. Robert is also present in the kitchen, talking with Gio. In response, both Gio, the selected next speaker, and Robert, the non-selected recipient, move to the stove and lean over to inspect the fries during a 2.0-second silence following the question. Ethnographically, Robert is said to be the cooking expert in the household. Also, he was positioned slightly closer to the fries, so arguably he could have responded more quickly. However, note that Robert does not immediately respond but defers to Gio as the selected next speaker. Furthermore, as soon as an answer is clearly projected, Robert initiates a move back to the counter. 
(13) Housemates 27:30.

1 Jud: Gio do you think these're done;

2 ((Gio and Rob both move to the stove) $) /(2.0)$

3 Gio: Oh [yeah.

4 Rob: $\quad[(($ turns from stove back to counter $))$

$5 \quad(0.2)$

6 Gio: Yeah. (totally) ((returns to counter))

Thus, both selected next speakers and non-selected recipients are oriented to the primary rights and obligations of selected next speakers to respond to questions that select them, as evidenced by the regularity of their respective responses and withholdings of responses at the transition relevance place.

There may be another level of orderliness in regard to how quickly a nonselected speaker responds (see Stivers 2001 for a discussion of this). With respect to more general interaction, it is certainly possible that people with closer relationships display this by responding in these environments, but this is beyond the scope of this article.

Evidence for a preference for selected next speakers to respond to questions. Looking at multi-party data provides insight into what sort of machinery underlies turn allocation. It suggests that AFTER a TRP, who self-selects becomes not rule-governed but preference-governed. One indication that after a transition relevance place there is a preference for the selected next speaker still to respond to the question is that, when non-selected recipients respond to questions that selected another as next speaker, they delay their responses until a normal transition space has passed (Stivers 2001). For instance, see (14). Here the selected next speaker provides an answer only after a substantial delay. In this case, several sorority housemates are having breakfast. Nancy had been about to leave the room when Celia asked her whether she was excited to meet a woman she had last seen when she was a child. As this sequence is closed, Sally asks, Who is it? Through both her gaze and the content of this question, she selects Nancy to provide an answer (Lerner 2003):

(14) Sorority Breakfast 2: 58:29

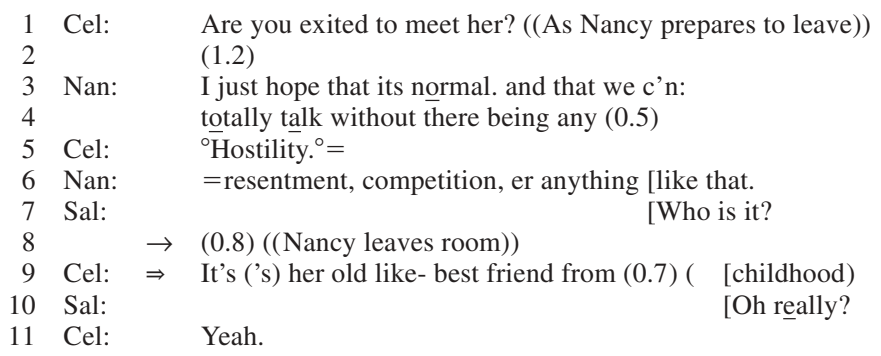

Nancy likely didn't hear the question in line 7, which was delivered from the other side of the room. Whatever the reason, Nancy leaves the room, and a si- 
lence of 0.8 seconds develops. Only at this point does Celia answer the question (line 9). In this context, this response is unproblematic. That is, like other dispreferred actions, it is delayed. With the delay, Celia orients to Nancy's primary rights as a selected next speaker. This case is extreme because if ever a nonselected speaker would have the right to answer a question, it would be when the selected speaker was unavailable as an interactant (e.g., no longer in the room). However, even here the response is delayed, underscoring the strength of this preference.

A similar example is shown in (15). Here, a family is having dinner. Cindy, the nine-year-old girl, has been telling about a field trip that she and her mother went on with her school to a local restaurant earlier that day. In line 1 , the father asks why the restaurant provided this service and fed all of the students and parents. Both through content and eye gaze, he selects Cindy to answer this question:

(15) Stew Dinner 12:10

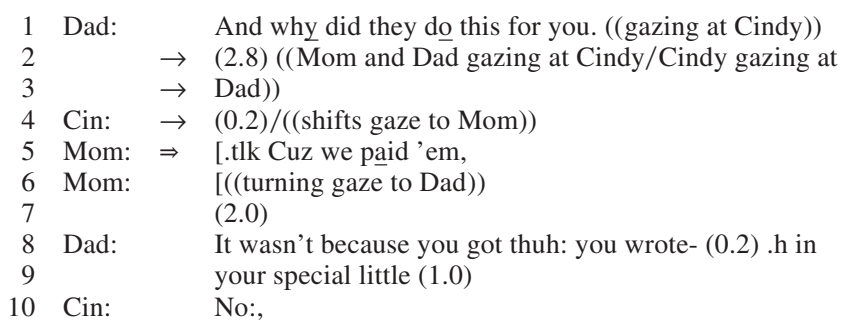

With their gaze and their silence, both the mother and the father treat Cindy as the selected next speaker - the one who appropriately should respond. It is only after a substantial silence (line 2) and, further, after Cindy shifts her gaze to her mother, that the mother provides an answer. The mother appears to understand Cindy's shift in gaze to her as a "hand-off" of the SPP to the mother to produce. These cases are directly in line with cases analyzed similarly in the pediatric context (Stivers 2001). Here the claim is that this is a more general interactional practice and not something based either in the medical context or in the parentchild context. Thus, there is further evidence that non-selected recipients are oriented to a response being both relevant and preferred by the selected next speaker. These cases show that beyond a normal transition space, non-selected recipients still orient to selected next speakers as having a primary right to respond. However, the data also suggest that after the normal transition space, non-selected recipients regularly answer questions. A question, then, is what nonselected recipients are doing producing an answer here, if it is a dispreferred action.

This brings us to the issue of a second order of organization among the two basic preferences that have been described thus far. Returning to the discussion of the turn allocation rule, we observed that at a transition relevance place, the 


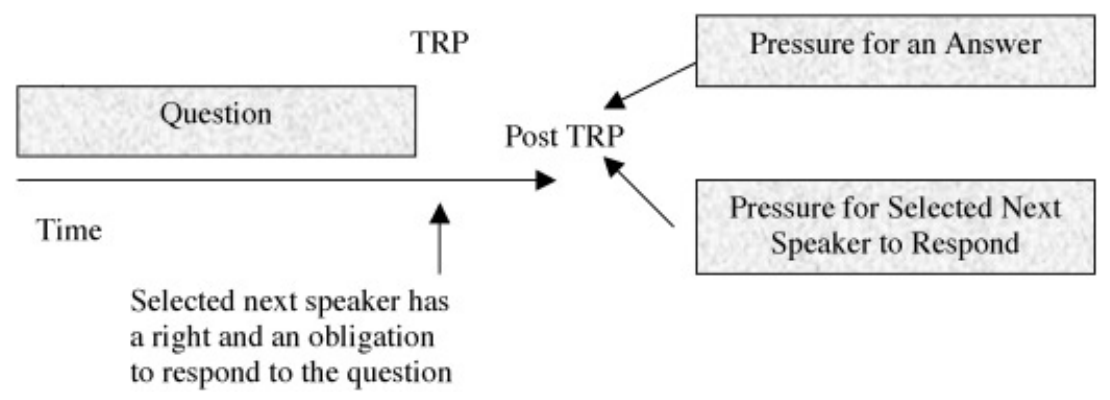

FIGURE 1: Diagram of turn allocation

rule appears to be treated AS A RULE. That is, if at that point a speaker has been selected, he or she, as specified by Sacks, Schegloff \& Jefferson 1974, has the right and obligation to speak next, and if another person does so, that person may be sanctioned. This sanctioning is critical to understanding this as a sort of rule-guided behavior. By contrast, once past the TRP, in a multi-party setting this rule may be conceptualized as being subject to two competing pressures, as shown in Figure 1. That is, once a normal transition space has occurred, there is both pressure for an answer to be provided over a non-answer response, and also pressure for a response to be provided by the selected next speaker. The final section of this article focuses on how these pressures are managed and on what systematicity exists for dealing with this problem.

PREFERENCE FOR ANSWER VS. PREFERENCE FOR THE

SELECTED NEXT SPEAKER TO RESPOND

There are three sequential environments in which non-selected recipients respond to questions: (i) when selected next speakers fail to respond at the TRP; (ii) when selected next speakers claim an inability to answer; and (iii) when selected next speakers vocally display difficulty in providing a definitive answer. These contexts support an analysis that interactants rank the preference for an answer higher than the preference for the selected next speaker to respond. This section will explore each of these sequential environments in turn. In all cases, what is of interest is how participants deal with the collision of these two preferences (the preference for an answer and the preference for the selected next speaker to respond). ${ }^{6}$

\section{When selected next speakers fail to respond at the transition relevance place}

As mentioned in the previous section, one way in which non-selected recipients respect the primary right of selected next speakers to take the next turn is by delaying their responses. However, once a response has noticeably not occurred 
(i.e., it was due but absent) and a gap has emerged, there is competing pressure to provide an answer as well. ${ }^{7}$ This is one of the main environments in which non-selected recipients respond, most commonly by answering (Stivers 2001). For instance, in (16), a family of four (Mom, Dad, and two children) are having dinner. When the phone rang several minutes earlier, the mother had proposed that they not answer it during dinner. The phone rang again a few seconds before the extract and was again answered by the answering machine. The father has just risen and is standing, leaning over the phone and answering machine in another part of the room. The mother selects the father to answer a question, using the address term Dad (line 1):

(16) Fish Dinner: 16:00

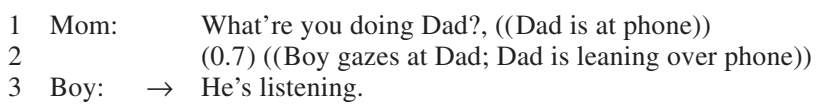

The question prefers an answer and makes relevant a response from Dad. However, over 0.7 seconds, Dad does not respond to the question. At this point a response from Dad is still preferred, but the pressure for an answer is competing. The boy has been gazing at Dad and thus is in a position at least to describe what he observes. He does this and provides an answer to the question with He's listening. The boy observes that Dad is attending to the phone and machine and is not projecting that a response is forthcoming during the 0.7 -second silence, and it is only at this point that the boy offers an answer to the question.

A second example of this type is shown in (17), which offers evidence that non-selected recipients provide not only preferred answers but also dispreferred answers. Non-selected recipients are probably just as oriented to the structural preferences for alignment as are selected next speakers. Furthermore, nonselected recipients may even be less inclined to provide disaligning answers because they are not obligated to respond in the way that the selected speaker is. Nonetheless, non-selected recipients do sometimes provide dispreferred answers. Below, Don and Jan have been talking even though they are part of a triadic interaction. At line 1, Don selects Jan to answer a question through his gaze. The question is a yes/no interrogative which is designed to prefer a "yes" answer. Jan does not immediately respond to the question, and a delay opens up in line 2. In this environment, Barbara, a non-selected recipient, provides an answer:

(17) MP3 42:41

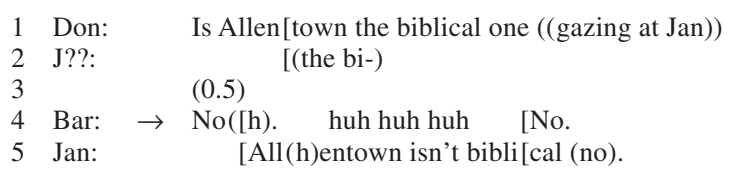


As mentioned earlier, this answer is dispreferred relative to the turn to which Barbara is responding. This offers evidence that non-selected speakers are concerned with the preference for providing an answer even if that means providing an answer that is itself not aligned and thus is dispreferred. Another example of this is shown in (18). Here several friends are hanging out together. Bates is off camera and therefore is either in another part of the room or in another room altogether. Stuart and Luke are sitting together around the breakfast bar in the kitchen. Stuart asks Bates if he has heard a story. Although this question is a vehicle for establishing story recipiency, a "yes" or "no" response is nonetheless relevant both for the question and as a go-ahead/block for the story. In response to the question, Bates does not immediately respond to the question, and a silence ensues (line 2). In this environment, similar to (17), a non-selected recipient provides an answer:

(18) SNS 51:36

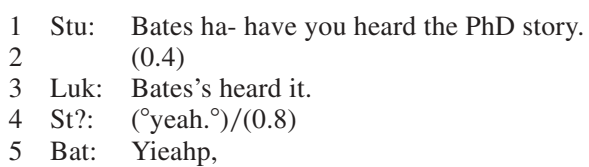

Also as in (17), this answer is dispreferred. Specifically, by claiming to know the story, Luke effectively blocks Stuart's telling of the story to Bates. Thus, both selected and non-selected recipients appear to be less inclined to produce dispreferred responses to questions - in this case, dispreferred answers. However, in environments where a selected next speaker has failed to respond immediately to a question, non-selected recipients display a preference for providing an answer over a preference for the selected next speaker to respond, even if that means producing a dispreferred SPP.

A final example is shown in (19). Three housemates are talking. At line 7, Judy asks Gio where he was doing pull-ups. In response, after some laughter and smiling, Lance, a non-selected recipient, provides an answer (line 9):

(19) Housemates 10:50

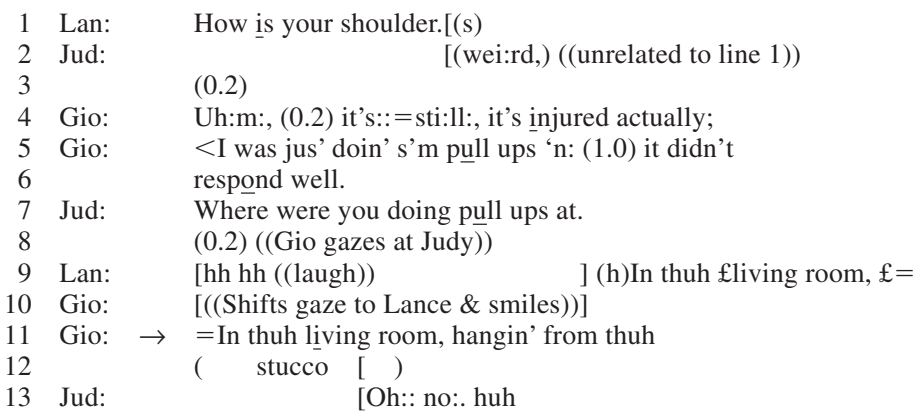


This case is also interesting because the answer provided is relatively nonspecific. In this sense, it might be considered a "non-type-conforming" (Raymond 2003) answer to the "where" question, since although it is superficially conforming by providing a location, what is most relevant is what Gio was hanging from. Thus, just as non-selected recipients provide dispreferred answers, they also provide nonconforming answers.

This section has focused on one common sequential environment in which non-selected recipients provide answers to questions that selected another as next speaker: when the selected next speaker has failed to respond at the TRP. This offers support for the claim that non-selected recipients are oriented to the primary rights of selected next speakers to respond but that after a TRP, they prioritize the preference for an answer over the preference for a response from the selected next speaker. A second common environment in which non-selected recipients provide answers to questions that selected another as next speaker is when selected next speakers claim an inability to answer.

When selected next speakers claim an inability to answer. On one hand, cases such as these pose no problem to the turn allocation rule, because the selected next speaker provides a response to the prior question and thereby addresses the relevance of an answer to the question; however, as discussed earlier, such nonanswer responses fail to promote the activity with which the question was concerned. Non-selected recipients orient to the outstanding relevance of an action that accomplishes this (most commonly, though not exclusively, an answer). Thus, the two preferences are in conflict here, just as they were when a gap emerged following the question. For instance, in (20), a pediatrician selects the six-yearold girl to respond to the question what's u:p. This question selects her through the repeating of her prior response, You're sick, (Schegloff et al. 1977, Lerner 2003), and then more explicitly with the question that follows, which uses this repetition as a resource for soliciting a specification of sick from the girl:

(20) P110; six-year-old girl

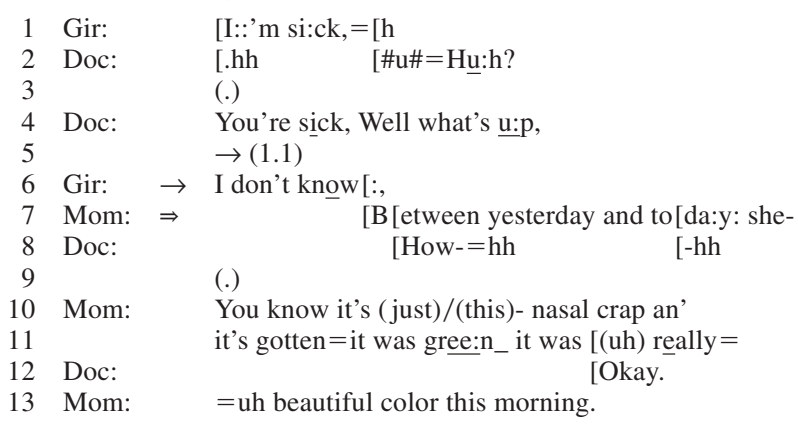

The mother does not respond initially, despite her clear ability to answer the question (see lines 7-10). Following a rather lengthy 1.1-second silence, the girl too displays her understanding that a response is due from her with her claim of 
inability to answer (line 6). Unlike in a context where the selected speaker readily provides an answer, in this case-as the silence emerges and then when the girl provides a non-answer response-two preferences can be seen to converge and conflict. Specifically, there is a preference for an answer as well as a preference for the response to be from the selected recipient, the girl. When the girl provides a non-answer response, the mother displays her orientation to the preference for an answer as overriding the preference that the response come from the selected recipient. However, the facts that a response was provided by the girl and that the mother delayed her turn in the service of getting that response nonetheless display the participants' orientations to the girl as having primary rights to speak next, and thus as being the preferred responding party.

A similar example is shown in (21). Several men are talking. Mike asks Sonny why he did not take the umbrella with him earlier when he apparently got rained on (line 5). In response, Sonny projects a response with the preface Ohweh- (line 6) but cuts this off. He then provides a non-answer response, I don'know (line 7). At this point, Rick answers the question: Wasn' raining when 'e left (line 8). ${ }^{8}$

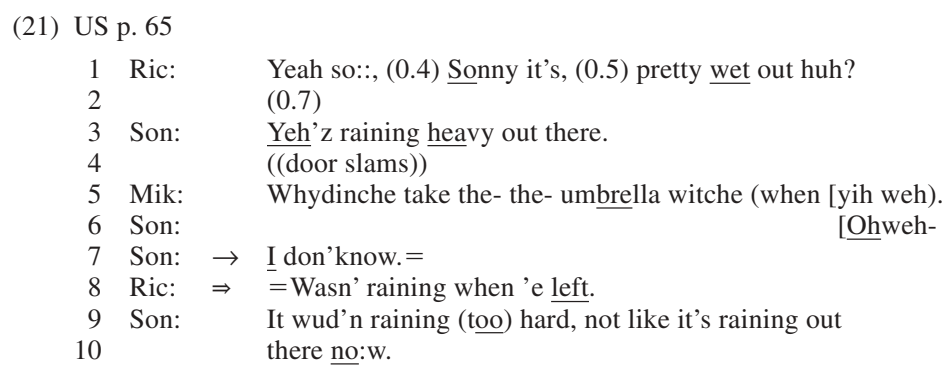

Similar to (20), here the non-selected recipient provides an answer once the selected next speaker has affirmatively failed to provide an answer. As mentioned above, this provides evidence that the preference for an answer overrides the preference for a response from the selected speaker. ${ }^{9}$ These cases suggest that when a selected next speaker responds but fails to provide an answer, nonselected recipients orient to the ongoing relevance and preference for an answer in response to the question.

This section has shown a second environment in which non-selected recipients provide answers to questions that selected another as next speaker. In this section it was again observed that non-selected recipients orient to the primary rights of selected next speakers to respond to questions that selected them. However, the fact that they offer answers after a non-answer response reflects a secondorder ranking of a preference for answers over a preference for selected next speakers to respond, once past the TRP.

A final environment in which non-selected recipients provide answers is when a selected recipient is displaying observable difficulty in providing an answer to 
the question. In these cases, the collision of preferences is particularly striking because the selected recipient is indeed responding to the question, but the nonselected recipient appears oriented not only to this as an action but also to the content of the response.

When the selected next speaker vocally displays difficulty in answering the question. Selected next speakers sometimes display difficulty in answering a question by hesitating or with markers of doubt. This projects that the production of an answer is in some way problematic for the selected next speaker. This is the third environment in which non-selected recipients can be observed to provide answers to questions that selected another as next speaker. In (22), for instance, Darci selects Brenda to answer a question in line 1. The question inquires about whether the house fax machine would need to be turned on to receive a fax:

(22) Sorority Breakfast 1: 47:33

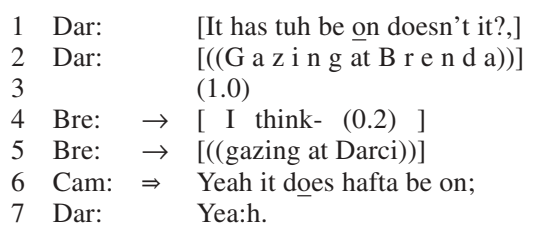

The selection of Brenda is done with Darci's gaze in combination with the question (Lerner 2003). However, following this, there is a 1.0-second silence. Brenda was oriented to her responsibility as the selected speaker by gazing back at Darci and beginning a response with I think- (line 4). However, this is cut off and then another delay begins, reaching 0.2 seconds. At this point Brenda has begun to respond and has projected that an answer will be forthcoming, but she also projects difficulty producing this answer with the multiple delays (both between-turn and within-turn) and with the cut-off of I think- - already an epistemic downgrade of the projected answer. With this cut-off, it is unclear whether she will abandon this effort to answer in favor of another action or will reformulate. It is here that Cammie provides an answer (line 6). Similar to cases where selected speakers provide non-answer responses, in contexts where selected speakers are having difficulty providing answers, this suggests that non-selected speakers are oriented to the competing pressure to provide an answer. Further, non-selected recipients appear to treat the preference for an answer as taking priority over the preference for the selected next speaker to respond if that response is a problematic one (e.g., not an answer or not definitive as an answer).

A similar response comes from (23), a neighboring sequence in the same data. Here, Ana selects Darci to answer a question (line 4). There is a delay (line 6), and then Darci projects a response but further delays her turn with Uhm and further with the stretch on the $U h:: m$. It is at this point that Brenda responds: 
(23) Sorority Breakfast 1: 47:24

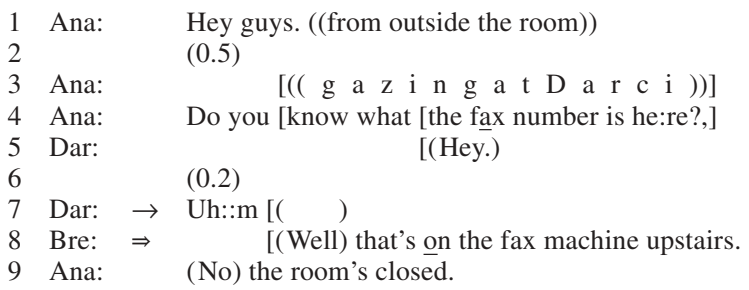

Here the response provided is not an answer. However, it does the work that answers generally do: It promotes the activity with which the question is concerned - locating the number. Thus, in contrast to non-answer responses, this responsive action not only completes the technical sequence but also promotes the activity insofar as it tells Darci where the number can be found. Thus, even in the rare cases where non-selected recipients do not answer, their responses orient to promoting the progress of the activity with which the question was concerned.

Each of the environments that has been discussed suggests that non-selected recipients maintain an orientation to the primary rights and obligations of selected next speakers to respond to questions. This has been evidenced in the environments in which they do respond by the observations that non-selected recipients delay their responses until after the transition space has passed and a selected speaker has displayed an inability to answer or has displayed difficulty in answering.

\section{I S C US S I O N}

We have shown that interactants display a systematic preference for answers over non-answer responses in response to information questions, and that they display a preference for selected next speakers to respond to questions over nonselected recipients. Most important, though, we asked whether and how participants deal with situations in multi-party contexts where, following a normal transition space, these two preferences collide. Here there is competing pressure for an answer and for the selected next speaker to respond. In these environments, this analysis revealed a systematic preference for the provision of an answer over the preference for the selected next speaker to respond.

This ordering of preferences suggests that interactants are concerned with advancing in-progress activities through sequences. That is, non-selected recipients appear to orient to the primary rights of selected next speakers to advance the action import of the sequence, but failing this, they appear to treat it as preferable to further the progress of the sequence themselves rather than to delay this progress in favor of the selected next speaker's doing so (or possibly still failing to do so). The last cases offered in the analysis section suggested this as well, because they showed that this concern with progressivity went beyond cases 
where there was no response projected or where a non-answer had been provided. These cases suggested that even when selected speakers display an orientation to responding, if they are having difficulty providing a response that furthers the progress of the activity (i.e., an answer), and this is past a transition space, then non-selected recipients themselves do work to promote progressivity.

This is very much in line with earlier conversation analytic findings which suggest that interactants are concerned with the progress of talk in interaction. For instance, Schegloff 1979 showed for repair that there is a preference for progressivity within a speaker's turn such that, in successive repairs, each repair initiation and resolution can be seen to further the production of the turn. When this ceases, it is not uncommon for the speaker to abandon the turn and reformulate. Similarly, within a turn, participants have been shown to be concerned with progressivity in terms of word searches. Goodwin \& Goodwin 1986 showed that, within a TCU, if a participant is having difficulty finding a word, other participants will provide candidate words which allow the turn to progress.

In terms of sequence progressivity, Schegloff states: "Sequences are the vehicle for getting some activity accomplished, and that response to the first pair part which embodies or favors ACCOMPLISHMENT OF THE ACTIVITY is the favored - or preferred - second pair part" (in press; emphasis added). Thus, in terms of sequence organization, preferred responses have generally been analyzed as actions which more quickly and efficiently allow for sequence closure and thus for progress in interaction. Schegloff 1979 further observed that there might also be a preference for progressivity in terms of putting the sequentially implicated next items "next" (e.g., for questions, this would be answers or nonanswer responses) rather than something like a repair sequence or other type of insert sequence (Schegloff, in press). This article furthers these arguments by relying on a second-order preference organization to reveal that, when problems in activity progress arise, participants have methods for dealing with this systematically. Here, non-selected recipients promote an in-progress activity when a selected next speaker has displayed difficulty in doing this himself or herself.

However, sequence progressivity is not only a structural issue. It is very much a social issue. The rules underlying the organization of interaction, such as the ones focused on here - the conditional relevance of a response to a question, and the conditional relevance of a response by a selected next speaker - are also resources by which persons' rights are maintained or violated. The structural process discussed here, then, is also about the rights of the interactants involved: the questioner and the questioned. Just as one preference can be observed to be prioritized over another, the rights of one speaker can be seen to be prioritized as well. We suggest that interactants display a preference for sequence and activity progressivity. But through this, they also prioritize the rights of questioners or sequence initiators. Consider that whatever activity an addressee is involved in, if a question selects that person, he or she can be sanctioned for not responding. Interventions by non-selected recipients may work specifically to deal with some 
of this inequity. For instance, in (16), the mother asks the father a question while he is attempting to listen to the answering machine. Responding to the question would undermine the listening activity. The boy's intervention with a response maintains the right of the questioner to an answer while maintaining the addressee's right to complete his in-progress activity.

It is likely that the level discussed here is one rather gross level of orderliness, and that far more granular levels are also present, though they could not be discussed here. For instance, (18), along with other cases in the larger corpus, suggests the possibility that when a non-selected speaker provides what Raymond 2003 identifies as non-conforming answers, previously selected recipients may be more likely to answer the question subsequently even if they too will provide non-conforming answers. Although too few cases were observed to confirm this, the data so far certainly support this possibility. Thus, this might be another way that non-selected recipients work to manage the conflict between the preference for progressivity and the preference for the selected speaker to respond.

We suggest that a concern for progressivity is not restricted to certain contexts (such as institutional contexts) nor to certain participants. For instance, adults could be seen answering for children and children answering for adults. However, it is likely that there is, again, more orderliness at the level of who responds if, in multi-party contexts, more than one respondent has the ability to provide an answer, for instance. Thus, it could be expected that family members, couples, and close friends might display their closeness through their ability to answer questions that selected their significant others. However, this remains to be investigated in its own right.

Similarly, although there has long been a mandate to explore interactional practices systematically across typologically and areally diverse languages (Levinson 1983, Brown \& Levinson 1987), little comparative work has been done. Levinson (2000:xiv) speculates that "there is obvious cultural codification of many aspects of language from phoneme to syntactic construction, whereas the uncodified, unnoticed, low-level background of usage principles or strategies may be fundamentally culture-independent." The concept of preference may be a rich area for such an investigation. For instance, do the same preferences exist cross-linguistically? And if so, when they come into conflict in other cultural and linguistic groups, is the preference for progressivity the preferred outcome? Specifically, cultural constraints might be such that in some communities, answering "for" other individuals would be a violation of their social norms and thus would not occur even when the selected individual has claimed an inability to answer the question. Ochs's work in the Samoan community reflects an analysis that in this highly stratified culture, high-status individuals will not attempt to interpret a problematic utterance spoken by a lower-status individual (Ochs \& Schieffelin 1984). From this it could be hypothesized that when a question selects a next speaker in multi-party conversation, the stratification would affect the preference ordering in one of two ways: In a 
conversation where a lower-status person was selected to respond in front of a higher-status person, the higher-status person might be less likely to answer on behalf of the lower-status person if this could be understood as contradicting the norm that the burden for clarification lies on the lower-ranking individual; alternatively, a lower-status person might be unwilling to speak on behalf of a higher-ranking individual because it might be perceived as disrespectful. Thus, whether and how such cultural constraints affect this and other levels of interactional organization needs further systematic investigation.

\section{A P P E N D I X}

Transcription Conventions

1. Temporal and sequential relationships

A. Overlapping or simultaneous talk is indicated in a variety of ways.

[ Separate left square brackets, one above the other on two successive

[ lines with utterances by different speakers, indicates a point of overlap onset, whether at the start of an utterance or later.

] Separate right square brackets, one above the other on two successive

] lines with utterances by different speakers indicates a point at which two overlapping utterances both end, where one ends while the other continues, or simultaneous moments in overlaps which continue.

$=\quad$ B. Equal signs ordinarily come in pairs - one at the end of a line and another at the start of the next line or one shortly thereafter. They are used to indicate two things:

(1) If the two lines connected by the equal signs are by the same speaker, then there was a single, continuous utterance with no break or pause, which was broken up in order to accommodate the placement of overlapping talk.

(2) If the lines connected by two equal signs are by different speakers, then the second followed the first with no discernible silence between them, or was "latched" to it.

(0.5) C. Numbers in parentheses indicate silence, represented in tenths of a second; what is given here in the left margin indicates $5 / 10$ seconds of silence. Silences may be marked either within an utterance or between utterances.

(.) D. A dot in parentheses indicates a "micropause," hearable but not readily measurable; ordinarily less than 0.2 second.

2. Aspects of speech delivery, including aspects of intonation.

A. The punctuation marks are not used grammatically, but to indicate intonation.

The period indicates a falling, or final, intonation contour, not necessarily the end

? of a sentence. Similarly, a question mark indicates rising intonation, not

, necessarily a question, and a comma indicates "continuing" intonation, not necessarily a clause boundary. A combined question mark and comma, which

?, indicates a rise stronger than a comma but weaker than a question mark.

- An underscore following a unit of talk indicates level/unchanging intonation.

; The semicolon indicates that the intonation is equivocal between final and "continuing"

: : $\quad$ B. Colons are used to indicate the prolongation or stretching of the sound just preceding them. The more colons, the longer the stretching.

C. A hyphen after a word or part of a word indicates a cut-off or self-interruption.

word D. Underlining is used to indicate some form of stress or emphasis, either by increased loudness or higher pitch. The more underlining, the greater the emphasis.

- E. The degree sign indicates that the talk following it was markedly quiet or soft. 
3. Other markings.

F. When two degree signs surround talk it indicates that all talk inside was quiet.

G. The circumflex symbol indicates a rise in pitch.

H. The combination of "more than" and "less than" symbols indicates that the talk between them is compressed or rushed. Used in the reverse order, they can indicate that a stretch of talk is markedly slowed or drawn out.

The "less than" symbol by itself indicates that the immediately following talk is "jump-started," i.e., sounds like it starts with a rush.

I. Hearable aspiration is shown where it occurs in the talk by the letter $h$ - the more $h$ 's, the more aspiration. The aspiration may represent breathing, laughter,

etc. If it occurs inside the boundaries of a word, it may be enclosed in parentheses in order to set it apart from the sounds of the word. If the aspiration is an inhalation, it is shown with a raised dot before it.

\# J. A number sign indicates gravelly voice quality on the sound(s) that follows or that falls between two number signs.

K. The British pound sign indicates "smile voice."

(( )) A. Double parentheses are used to mark transcriber's descriptions of events, rather than representations of them, e.g. ((cough)), ((sniff)), ((telephone rings)), ((footsteps $)),(($ whispered $)),(($ pause $))$.

(word) B. When all or part of an utterance is in parentheses, or the speaker identification is, this indicates uncertainty on the transcriber's part, but represents a likely possibility.

( ) Empty parentheses indicate that something is being said, but no hearing (or, in some cases, speaker identification) can be achieved.

\section{NOTES}

*Thank you to Nick Enfield, Steve Levinson and Manny Schegloff for useful discussions about the phenomena discussed in this article, and to John Heritage for his careful reading and comments on earlier drafts. Portions of this article were presented at the 2002 Western States Communication Association convention, Long Beach, California, and at the Workshop on Feedback in Interaction at the Max Planck Institute for Psycholinguistics, Nijmegen, in February 2004.

${ }^{1}$ It is relatively uncommon for non-selected recipients to perform other actions. Of the cases where this occurred, one primary action speakers were performing was prompting the selected speaker (e.g., You've gotta tell him) to provide an answer or to do teasing; for example, following a question about trying the peas during dinner, a non-selected recipient quips They're good for you.

${ }^{2}$ Clayman 2002 asserts that there is a preference for answers over non-answers but offers little evidence for the claim. Primarily, he suggests that non-answers are accountable. Because much of the later analysis in this article rests on this claim, this section is concerned to offer substantially more evidence for this claim.

${ }^{3}$ There may be additional factors that shape whether or not a recipient of a question provides a response that tries to answer when answering is difficult or impossible. Accountability for knowing is one such factor. However, this is beyond the scope of this article.

${ }^{4}$ Note that other actions that are not concerned with responding to the question are done as intervening actions by non-selected recipients, although following questions these are very rare. We are not attempting here to address these types of actions (for a discussion of this phenomenon, see Lerner 2002).

${ }^{5}$ Note that the frequencies reported total $97 \%: 85 \%$ of the time selected speakers provide answers, $12 \%$ of the time selected speakers provide some other response, and $3 \%$ of the time either no response is provided or a non-selected recipient provides some response.

${ }^{6}$ The concept of preferences coming into conflict has been discussed by Schegloff (in press) in terms of "cross-cutting preferences." However, this term has been used specifically to refer to cases where the preference of the action (e.g., an accusation) conflicts with the preference embodied by the turn design - the vehicle for that action (e.g., the question). In this context, Schegloff observes 
that the preference of the action typically overrides the preference of the vehicle for that action. Here, the concern is with a different sort of conflict among preferences, and the issue is with the second-order organization of the two types of preferences (Sacks \& Schegloff 1979).

${ }^{7}$ To be clear, the turn allocation rule does not at any time cease to be relevant. That is, it does not appear that a response by a non-selected recipient ever becomes CONDITIONALLY RELEVANT. For this to be the case, a prior speaker would have to select a new next speaker, for instance by shifting gaze from one person to another. Moreover, if neither a selected next speaker nor a non-selected recipient provides an answer, regardless of the time that has elapsed, the selected next speaker CAN be held accountable for this failure, whereas a non-selected speaker cannot. Thus, when speaking of two competing preferences, the implication is that the rule is incrementally relaxed, suggesting that an additional order of organization exists past the TRP which is laminated on top of the existing rule.

${ }^{8}$ In both this case and in the case shown as (1) and (20), there is evidence that non-selected recipients may "answer the question" while specifically not dealing with the action import of the question (e.g., the accusation).

${ }^{9}$ Some might argue only a selected next speaker could truly answer this question, but the pressure for an answer nonetheless appears to place substantial pressure on the non-selected interactants to yield an answer.

\section{REFERENCES}

Atkinson, J. Maxwell, \& Heritage, John (1984) (eds.). Structures of social action: Studies in Conversation Analysis. Cambridge: Cambridge University Press.

Beach, Wayne A., \& Metzger, Terri R. (1997). Claiming insufficient knowledge. Human Communication Research 23:562-88.

Brown, Penelope, \& Levinson, Stephen C. (1987). Politeness: Some universals in language usage. Cambridge: Cambridge University Press.

Clayman, Steven (1993). Booing: The anatomy of a disaffiliative response. American Sociological Review 58:110-30.

(2002). Sequence and solidarity. In E. J. Lawler \& S. R. Thye (eds.), Advances in group processes: Group cohesion, trust and solidarity, 229-53. Oxford: Elsevier Science.

Golato, Andrea (2002). German compliment responses. Journal of Pragmatics 34:547-71.

Goodwin, Marjorie Harness, \& Goodwin, Charles (1986). Gesture and coparticipation in the activity of searching for a word. Semiotica 62:51-75.

Heritage, John (1984). Garfinkel and ethnomethodology. Cambridge: Polity.

(1988). Explanations as accounts: A conversation analytic perspective. In C. Antaki (ed.), Understanding everyday explanation: A casebook of methods, 127-44. Beverly Hills, CA: Sage.

Lerner, Gene (2003). Selecting next speaker: The context sensitive operation of a context-free organization. Language in Society 32:177-201.

(2002). Practice does not make perfect: Intervening actions in the selection of next speaker. Paper presented at the UCLA Conference on Language, Interaction, and Culture, Los Angeles, California.

Levinson, Stephen C. (1983). Pragmatics. Cambridge: Cambridge University Press. (2000). Presumptive meanings: The theory of generalized conversational implicature. Cambridge, MA: MIT Press.

Ochs, Elinor, \& Schieffelin, Bambi B. (1984). Language acquisition and socialization: Three developmental stories and their implications. In R. Shweder \& R. LeVine (eds.), Culture theory: Essays on mind, self and emotion, 276-320. New York: Cambridge University Press.

Pomerantz, Anita (1978). Compliment responses: Notes on the co-operation of multiple constraints. In J. Schenkein (ed.), Studies in the organization of conversational interaction, 79-112. New York: Academic Press.

(1984a). Agreeing and disagreeing with assessments: Some features of preferred/dispreferred turn shapes. In Atkinson \& Heritage (eds.), 57-101.

(1984b). Pursuing a response. In Atkinson \& Heritage (eds.), 152-64.

Raymond, Geoffrey (2003). Grammar and social organization: Yes/no interrogatives and the structure of responding. American Sociological Review 68:939-67. 
Sacks, Harvey (1967). Next-speaker selection techniques; Paired utterances. Paper presented at the Lectures on Conversation, Oxford, UK. (1973a). Lectures at the Summer Institute of Linguistics, Ann Arbor. (1973b). The preference for agreement in natural conversation.

(1987a). On the preferences for agreement and contiguity in sequences in conversation. In G. Button \& J. R. E. Lee (eds.), Talk and social organisation, 54-69. Clevedon, UK: Multilingual Matters.

(1987b). 'You want to find out if anybody really does care'. In Button \& Lee (eds.), Talk and social organisation, 219-25.

Sacks, Harvey, \& Schegloff, Emanuel A. (1979). Two preferences in the organization of reference to persons and their interaction. In G. Psathas (ed.), Everyday language: Studies in ethnomethodology, 15-21. New York: Irvington.

Sacks, Harvey; Schegloff, Emanuel A.; \& Jefferson, Gail (1974). A simplest systematics for the organization of turn-taking for conversation. Language 50:696-735.

Schegloff, Emanuel A. (1972). Notes on a conversational practice: Formulating place. In D. Sudnow (ed.), Studies in social interaction, 75-119. New York: Free Press.

(1979). The relevance of repair for syntax-for-conversation. In T. Givon (ed.), Syntax and semantics 12: Discourse and syntax, 261-88. New York: Academic Press.

(in press). A primer for conversation analysis: Sequence organization. Cambridge, England: Cambridge University Press.

; Jefferson, Gail; \& Sacks, Harvey (1977). The preference for self-correction in the organization of repair in conversation. Language 53:361-82.

\& Sacks, Harvey (1973). Opening up closings. Semiotica 8:289-327.

Stivers, Tanya (2001). Negotiating who presents the problem: Next speaker selection in pediatric encounters. Journal of Communication 51(2):1-31.

(Received 18 October 2004; revision received 27 June 2005; accepted 2 July 2005; final revision received 13 July 2005) 